\title{
Efficacy of a New Post-Mouthwash Intervention (Wiping Plus Oral Nutritional Supplements) for Preventing Aspiration Pneumonia in Elderly People: A Multicenter, Randomized, Comparative Trial
}

\author{
Takashi Higashiguchi Hiroshi Ohara Yayoi Kamakura Takeshi Kikutani \\ Masafumi Kuzuya Hiromi Enoki Hiromi Sanada Masami Matsuzaki \\ Michio Maruyama \\ Department of Surgery and Palliative Medicine, Fujita Health University School of Medicine, Toyoake, Japan
}

\author{
Keywords \\ Aspiration · Elderly · Mortality · Nursing home · Oral \\ nutritional supplements · Oral care · Wiping · Pneumonia · \\ Swallowing impairment
}

\begin{abstract}
Background/Aims: Aspiration pneumonia is a common cause of death among the elderly ( $\geq 90$-year-old) in nursing homes. Studies suggest that its incidence could be reduced by oral care interventions. We aimed to evaluate the efficacy of a new oral care intervention: wiping plus oral nutritional supplements (ONS). Methods: This prospective observational study was conducted in 252 patients (age $88.0 \pm 6.5$ years) in 75 nursing homes, rehabilitation hospitals, and other care facilities. Patients were randomly divided into an intervention group ( $n=74)$ and a control group $(n=107)$, whose members received conventional oral care. Body mass index, activities of daily living (Barthel index), and complete blood count and biochemistry parameters were measured at 2, 4, 6, and 8 months. Results: The cumulative incidence of pneumonia at 8 months tended to be lower in the intervention than in the control group (7.8 vs. $17.7 \%, p=0.056)$ and was significantly lower for men in the intervention group ( $p=0.046)$.
\end{abstract}

Conclusions: Our new intervention "wiping plus providing ONS" method appears to help prevent aspiration pneumonia, thereby reducing mortality risk. In this study, we disseminate information on how this method is used in Japan.

(c) 2017 S. Karger AG, Basel

\section{Introduction}

According to a 2011 annual report in Japan, pneumonia is the third leading cause of death overall and the leading cause of death in the very elderly; most pneumoniaassociated deaths in the very elderly are attributed to aspiration pneumonia. Aspiration pneumonia is believed to develop in patients with dysphagia after aspirating oral bacteria together with saliva or food. According to the 2011 Report of the Research Program on Eating Disorders and Dysphagia (led by the National Center for Geriatrics and Gerontology) [1], more than $40 \%$ of elderly Japanese patients in medical and long-term care facilities are affected by eating disorders or dysphagia. As Japan has become a so-called super-aging society, eating disorders or dysphagia should be a major challenge among elderly people year by year. Thus, national health expendi-

\section{KARGER}

(c) 2017 S. Karger AG, Basel

E-Mail karger@karger.com

www.karger.com/anm
Takashi Higashiguchi, $\mathrm{MD}, \mathrm{PhD}$

Department of Surgery and Palliative Medicine

Fujita Health University School of Medicine

1-98 Dengakugakubo, Toyoake, Aichi 470-1192 (Japan)

E-Mail t-gucci3021@herb.ocn.ne.jp 
tures are likely to grow with the necessity of treatment for aspiration pneumonia; actively taking measures to prevent aspiration pneumonia is extremely important for future medical care in Japan.

Recently, several important measures to prevent aspiration pneumonia have been recognized: providing oral care, rehabilitation for the ingesting/swallowing function, and improving nutrition.

Ikeda et al. [2] compared 3 oral care methods for their efficacy in reducing oral bacterial counts. They studied healthy adults, who were divided into 3 groups: those receiving oral care (1) with wet wipes for mouth cleaning (the "wiping" method); (2) using a water-injection cleaning device; and (3) with a sponge brush. The study demonstrated that the wiping method was the most effective for removing oral contaminants.

In another study conducted by the same group [3], the efficacy of wiping versus water injection as cleaning methods was compared in 31 elderly patients at nursing care facilities. The result showed that there was no difference between the 2 methods. Because wiping after using mouthwash is easier than water injection, this method may be a useful strategy to prevent the onset of aspiration pneumonia.

Administering oral nutritional supplements (ONS) to elderly people or patients with a disease-associated malnutrition has recently attracted attention. According to the guidelines of the European Society of Clinical Nutrition and Metabolism [4], ONS improved malnutrition in the elderly [4]. According to a meta-analysis of 9 randomized, comparative trials ( $n=1,190$ participants) [5], ONS reduced the readmission rate of 834 elderly subjects aged $\geq 65$ years who participated in 6 trials. In another study of 77 dementia patients at nursing care facilities [6], ambulatory ability and grip strength were well maintained in 42 patients assigned to the group receiving ONS.

In another meta-analysis in dementia patients at longterm care facilities, physical parameters - for example, body weight, body mass index (BMI), cognitive ability improved more significantly in the group receiving ONS $(n=1,076)$ than in the control group $(n=748)$ [7].

Some reports have also demonstrated the cost-effectiveness of administering ONS. That survey showed that (1) medical costs were reduced by $21.6 \%$ (USD 4,734 per patient), (2) hospitalization stays were shortened by an average of 2.3 days, and (3) the rate of readmission within 30 days was reduced by $2.3 \%$.

Thus, it is a very important trial for the prevention of aspiration pneumonia; a new oral care, including wiping with ONS, is performed on elder people and with this in mind, a prospective, randomized, comparative study was

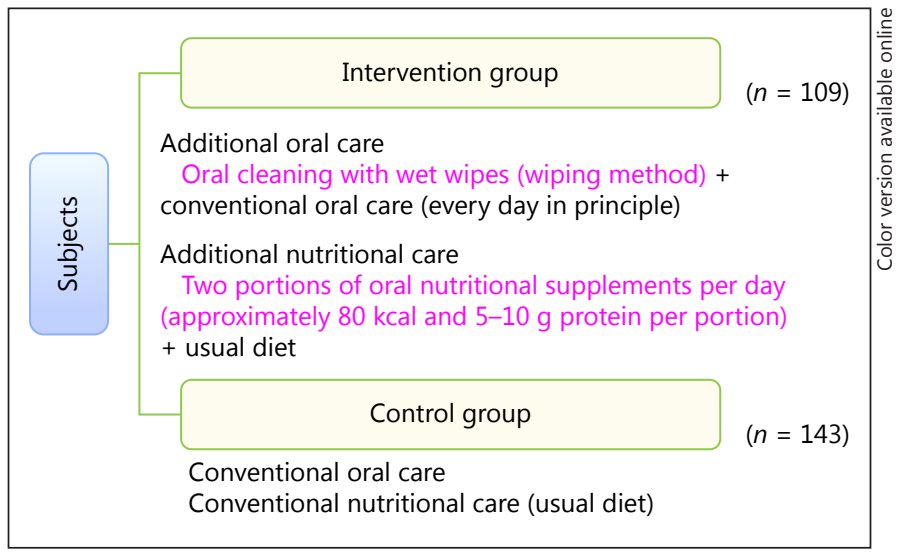

Fig. 1. Study design.

conducted to evaluate the efficacy of a new oral care intervention (i.e., wiping plus providing ONS) for preventing aspiration pneumonia in a high-risk population of elderly people in medical and long-term care facilities in whom swallowing function and nutritional status had deteriorated.

\section{Subjects and Methods}

\section{Subjects}

Regarding an association between the onset of pneumonia and the oral environment in elderly patients requiring nursing care, we referred to findings from the report.

Based on the aforementioned reports, we included patients who were likely to be at high risk for aspiration pneumonia and (1) were $\geq 75$ years at the time of consent $[1,8]$; $(2)$ had a BMI $<18.5$ $\mathrm{kg} / \mathrm{m}^{2}$ [9]; (3) had a serum albumin level <3.5 g/dL [10]; (4) were with dysphagia but had capacity for oral food intake, and would need a thickening agent for drinks for $\geq 30$ min each meal [8]; (5) gave consent themselves or had consent given by their family or legal representative.

Patients with the following conditions were excluded: (1) whose life expectancy was expected to be $\leq 1$ year; (2) for whom a feeding tube was used; (3) who experienced the onset of pneumonia (or symptoms suspected of pneumonia) within 1 month of enrollment; or (4) who were considered inappropriate for participation as judged by the study director.

The present study was a prospective, observational study designed as a multicenter, block-randomized, parallel-group comparison study. Participating facilities were publicly solicited and then randomly assigned to provide either wiping plus ONS (intervention group) or conventional oral care (control group) using the envelope method for block and randomization in a centralized registration system (Fig. 1). A total of 266 subjects from 75 facilities were registered between December 2013 and May 2015. Ten were excluded before the start of the study, and 4 were later found to be ineligible. The remaining 252 subjects were finally enrolled in the present study. 


\section{Interventions}

In all facilities evaluating control subjects, the conventional oral care and diet provided by caregivers and dietitians at each facility were continued. In all facilities assigned to evaluate the intervention group, subjects received an oral care intervention (wiping) and were given ONS in addition to their usual oral care and diet.

\section{Additional Oral Care Intervention: Wiping}

Subjects in each facility assigned to the intervention group received oral care with wet wipes for mouth cleaning (wiping) to remove oral bacteria every day during the intervention period (in principle) in addition to conventional oral care that was provided by caregivers at each facility. The wet wipes used was the following commercial product: Refre Care W (Bean Stalk Snow Co., Ltd., Sapporo, Japan).

Additional Nutritional Care: ONS

Subjects in the intervention group received ONS (100-200 kcal/ day and 10-20 g protein/day) in addition to their normal diets. The following commercial ONS products were supplied to the facilities by the study: Inner Power (Otsuka Pharmaceutical Factory, Inc., Naruto, Japan); Isocal Jelly, Isocal Jelly HC, Isocal Jelly PCF, Isocal Support Jelly, PemPal Thickened (Nestlé Health Science Co., Ltd., Lausanne, Switzerland); Enjoy Cup Jelly (Clinico Co., Ltd., Tokyo, Japan); Terumeal Jelly (Terumo Corporation, Tokyo, Japan); Egao Jelly Dan-Dan-Egao (Asahi Kasei Pharma Co., Ltd., Tokyo, Japan).

Before starting the study, an explanatory meeting was held to provide an outline of the study to all participating facilities. For the facilities assigned to evaluate the intervention group, additional explanations were provided to ensure provision of intervention "wiping plus ONS", and a DVD was distributed to the personnel at each facility to explain the proposed oral care intervention.

Survey Items

Baseline Characteristics

For each participating facility, the following data were recorded: the type of facility, number of beds, and availability of assistance by a dentist or dental hygienist, and a national registered dietitian. As baseline characteristics, we recorded patients' gender, age, body height, body weight, history of vaccination against pneumococcus, history of pneumonia, caloric intake (as food), and protein intake.

\section{Food Intake}

The amount of calories served was recorded each day. In the facilities in the intervention group, ONS provided was also recorded.

Physical Measurements

Body height, body weight, and crural circumference were measured at baseline (immediately before starting the study), every 2 months thereafter, and at the end of the study.

Hematology and Blood Biochemistry Measurements

Hematology and blood biochemistry tests were performed immediately prior to starting the study (baseline) and at the end of the study at facilities where these examinations were practicable.

Onset of Complications

If pneumonia developed during the period from baseline until the end of the study, the date of onset, severity, outcome, and therapeutic measures taken were recorded. Diagnosis of aspiration

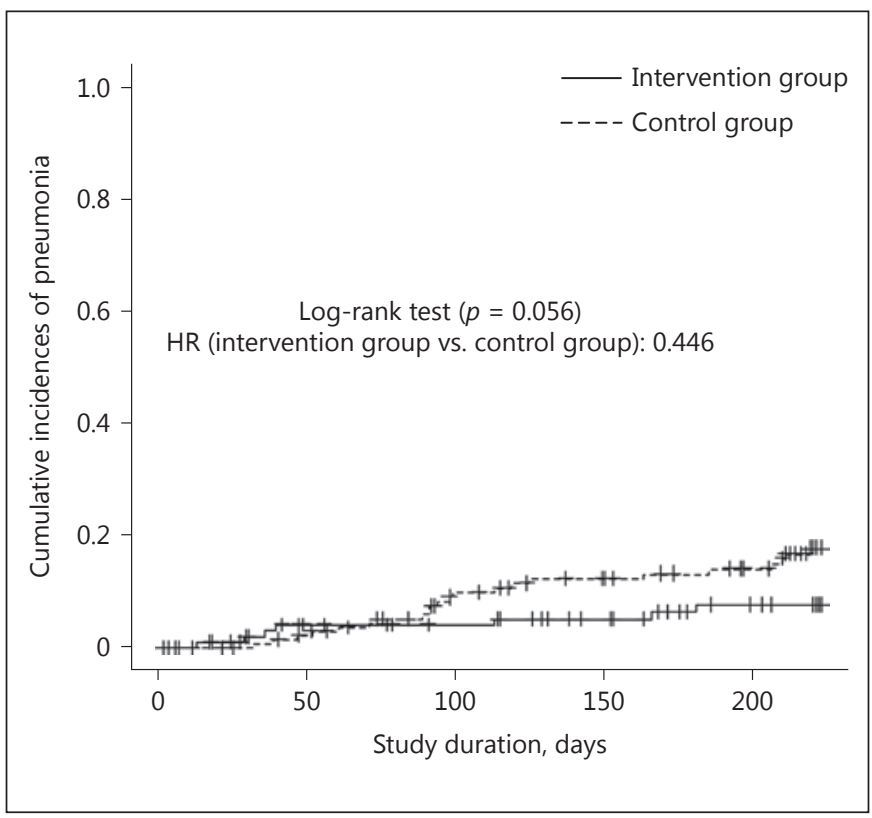

Fig. 2. Cumulative incidences of pneumonia (all subjects).

pneumonia was made by physicians in commissioned faculty based on X-ray images. If pyrexia of $37.5^{\circ} \mathrm{C}$ or higher occurred, the same items were recorded. If chronic obstructive pulmonary disease, chronic cardiac failure, or pressure sores occurred or worsened, the date of onset and severity of the disease were recorded.

\section{Statistical Analyses}

Esumi Co., Ltd., performed the data analyses. The cumulative incidence of pneumonia at various time points following the start of the study was defined as the primary endpoint. Inter-group differences were analyzed for the full analysis set using log-rank tests (significance level at 0.05). Student $t$ tests were performed to analyze intra-group and inter-group differences of the secondary endpoints. The mean daily caloric intake and mean daily number of oral care provisions during the entire study period were compared between the 2 groups.

\section{Results}

\section{Types of Participating Facilities}

Of the 75 participating facilities, 26 were assigned to the intervention group and 49 to the control group. In the intervention group, there were higher proportions of intensive care homes for the elderly and nursing care facilities for the elderly than rehabilitation hospitals and other facilities. On the contrary, the proportions of nursing care facilities for the elderly and other facilities were higher in the control group (Fig. 2). 
Table 1. Baseline characteristics of all subjects enrolled

\begin{tabular}{|c|c|c|c|}
\hline Baseline characteristics (category) & $\begin{array}{l}\text { Intervention group } \\
(n=109)\end{array}$ & $\begin{array}{l}\text { Control group } \\
(n=143)\end{array}$ & $p$ value \\
\hline Age, years & $88.3(5.8)$ & $87.9(7.0)$ & 0.573 \\
\hline Male & $20(18.3)$ & $34(23.8)$ & \\
\hline Female & $89(81.7)$ & $109(76.2)$ & \\
\hline Body weight at baseline & $36.2(6.0)$ & $37.5(6.6)$ & 0.098 \\
\hline Activities of daily living at baseline & $17.8(21.2)$ & $14.5(15.3)$ & 0.298 \\
\hline Albumin level & $3.15(2.18)$ & $2.83(0.94)$ & 0.137 \\
\hline \multicolumn{4}{|l|}{ History of pneumonia } \\
\hline Yes & $32(29.4)$ & $36(25.2)$ & 0.477 \\
\hline No & $77(70.6)$ & $107(74.8)$ & \\
\hline No & $20(18.7)$ & $36(26.1)$ & $0.024^{*}$ \\
\hline Yes & $67(62.6)$ & $60(43.5)$ & \\
\hline Potage-like & $9(8.4)$ & $15(10.9)$ & \\
\hline
\end{tabular}

$\%$, Fisher's exact test; mean, Student $t$ test; ${ }^{*} p<0.05$. There were no significant differences among the unasterisked data. Results are given as the mean (SD) or the number (\%) unless otherwise stated.

\section{Patient Baseline Characteristics}

For all subjects (54 men, 198 women), the mean age was $88.0 \pm 6.5$ years. No significant differences were observed between the intervention and control groups as described in Table 1. The subjects' swallowing function at baseline was relatively better in the control group than in the intervention group.

There were no significant differences between the genders. However, subgroup analyses of the intervention and control groups according to gender showed that the proportion of female subjects who have better swallowing function was significantly higher in the control group than in any other subject group.

\section{Cumulative Incidence of Pneumonia}

The cumulative incidences of pneumonia at month 8 were 7.8 and $17.7 \%$, respectively, for the intervention and control groups. Although the incidence of pneumonia was higher in the control group than in the intervention group $(p=0.056)$, this difference was not significant (Fig. 3).

Subgroup analyses according to gender for both groups showed no significant inter-group difference in the cumulative incidence of pneumonia in female subjects, whereas that in male subjects was significantly higher in

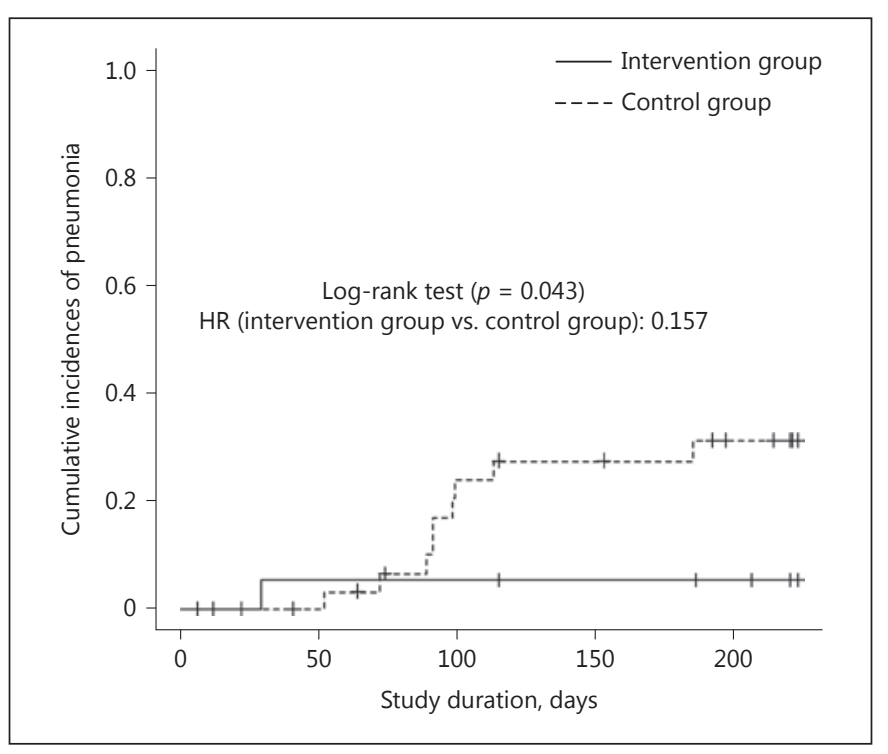

Fig. 3. Cumulative incidences of pneumonia (male).

the control group than in the intervention group ( $p=$ 0.046; Fig. 4a, b).

As seen in Table 2, among subjects in the intervention group from the rehabilitation hospital, the cumulative incidences of pneumonia were higher than those in the 
Table 2. Cumulative incidences of pneumonia at month 8 according to facility type

\begin{tabular}{|c|c|c|c|c|c|}
\hline Facility & $n$ & cumulative incidence, $\%$ & $n$ & cumulative incidence, $\%$ & $p$ value* \\
\hline Nursing care facility for the elderly & 49 & 7.7 & 40 & 15.3 & 0.296 \\
\hline Rehabilitation hospital & 11 & 22.2 & 12 & 9.1 & 0.360 \\
\hline Other & 0 & - & 32 & 26.8 & - \\
\hline
\end{tabular}

* log-rank test.

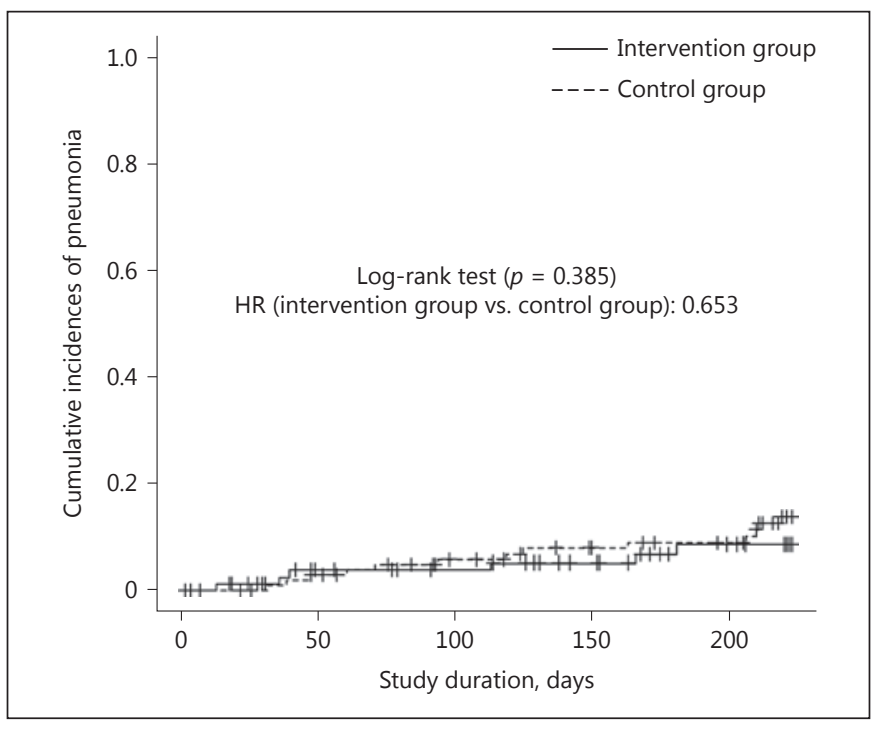

Fig. 4. Cumulative incidences of pneumonia (female).

control group. Another analysis was performed in a total of 197 subjects (108 from intensive care homes for the elderly, 89 from nursing care facilities for the elderly). The cumulative incidences of pneumonia were 6.3 and $15.6 \%$, respectively, in the intervention and control groups, and the difference was significant $(p=0.048$; Fig. 5).

\section{Secondary Endpoints}

Physical Measurements (Body Weight and Crural

Circumference)

Body weights were found to increase significantly from baseline only in the intervention group (Table 3 ).

Changes from the baseline body weight differed significantly between 2 groups at month $6(1.2 \pm 2.7$ and $0.02 \pm 2.2 \mathrm{~kg} ; p=0.011$ ).

Efficacy of a New Post-Mouthwash Intervention

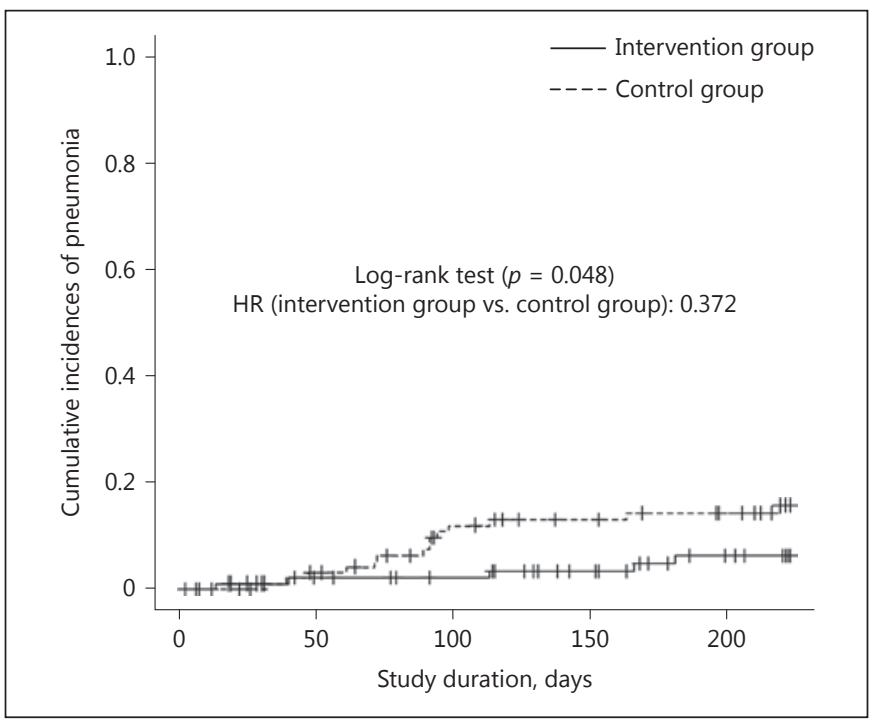

Fig. 5. Cumulative incidences of pneumonia (nursing care facilities + intensive care homes).

Changes in the crural circumference differed significantly between 2 groups at month $8(0.7 \pm 1.1$ and $-0.2 \pm$ $0.3 \mathrm{~cm} ; p=0.038)$.

\section{Blood Biochemistry}

There were no significant changes in hemoglobin, total protein, albumin, or total cholesterol levels from baseline to the end of the study (month 8 ) or between 2 groups. The total lymphocyte count at month $8(1,574.9 \pm$ $514.1 / \mu \mathrm{L})$ had increased significantly from baseline $(1,405.6 \pm 448.3 \mu \mathrm{L} ; p=0.029)$, and choline esterase level at month $8(216.4 \pm 63.6 \mathrm{mg} / \mathrm{dL})$ had also increased significantly from baseline $(203.8 \pm 51.2 \mathrm{mg} / \mathrm{dL} ; p=$ $0.021)$ in the intervention group.

No significant differences were observed between 2 groups with regard to all parameters (8 month). 
Table 3. Changes in secondary endpoints from baseline at different time points

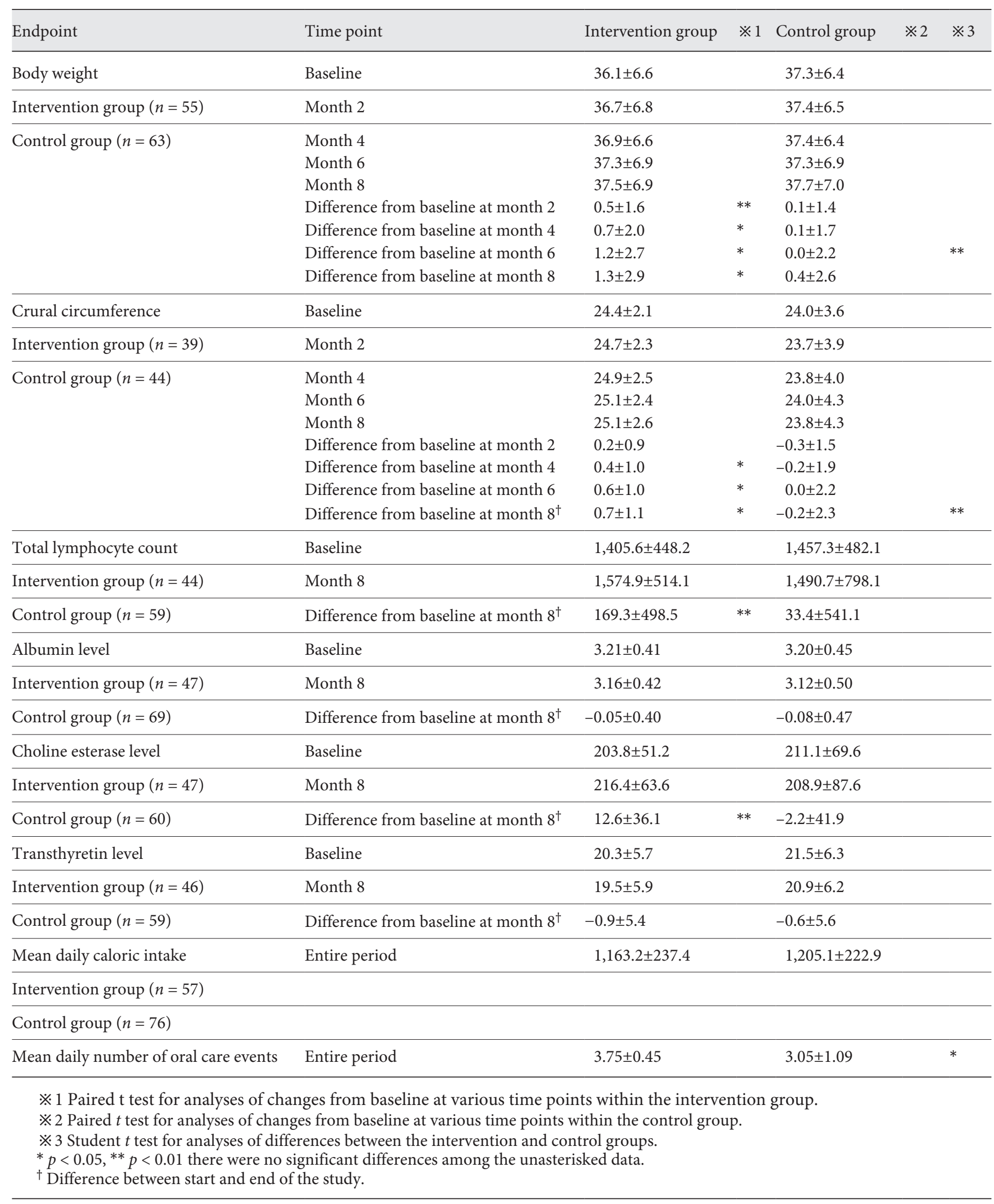


Mean Daily Caloric Intake

The mean daily caloric intakes for the total duration of the study were $1,163.2 \pm 237.4$ and $1,205.1 \pm 222.9 \mathrm{kcal}$, with no significant inter-group difference $(p=0.299)$, but the sufficiency rate was higher in the intervention group (95.6 vs. $91.2 \%)$.

\section{Discussion}

\section{Cumulative Incidences of Pneumonia}

We evaluated the efficacy of an unprecedented combination of wiping to remove oral bacteria after oral care and administering ONS, a simple and convenient means to compensate for insufficient daily food intake, for preventing aspiration pneumonia.

The results showed that the overall cumulative incidence of pneumonia tended to be higher in the control group than in the intervention group, although the difference was not statistically significant. This failure to demonstrate statistical significance was probably a result of the following conditions: The proportion of subjects who did not require any thickening agent for drinks was significantly higher in the control group than in the intervention group ( $p=0.024)$, suggesting that the control group had higher initial swallowing function; further, the proportion of female subjects who did not require any thickening agent for drinks was significantly higher in the control group than the intervention group $(p=0.009)$. Note, however, that the baseline characteristics of male subjects showed no inter-group difference. Because of the unbalanced distribution of baseline characteristic(s), more subjects with better swallowing function, and thereby physically less susceptible to aspiration pneumonia, were enrolled in the control group than in the intervention group. This could have resulted in no significant inter-group difference.

The subgroup analysis performed on the basis of gender showed that the cumulative incidence of pneumonia for the male subjects was significantly higher in the control group (31.2\%) than that in the intervention group $(5.3 \% ; p=0.046)$ despite no significant inter-group difference for female subjects.

In the present study, a significant reduction of the cumulative incidence of pneumonia (5.3\%) with wiping plus ONS was clearly demonstrated for male subjects.

The present oral care intervention may have prevented the onset of pneumonia in some high-risk female subjects who would otherwise have developed pneumonia. In contrast, there was no significant inter-group difference

Efficacy of a New Post-Mouthwash Intervention in the proportion of high-risk subjects at baseline for the men, suggesting that the more balanced distribution of risk factors between the 2 groups for male subjects could have contributed to the demonstrated efficacy of the present oral care intervention for men.

Based on the available data on differences between the genders regarding the incidence of pneumonia, there is no direct evidence that men are at higher risk for developing pneumonia. One report by Alan et al. [11], who studied the long-term prognosis in patients with community-acquired pneumonia, reported a higher mortality rate and more age-related sarcopenia in male than in female.

This result is also probably because of a high femaleto-male ratio in the study population; women accounted for $78.6 \%$ of all study subjects. The significant differences observed among male subjects suggest the efficacy of the present oral care intervention for preventing the onset of aspiration pneumonia.

The subgroup analyses of the incidences of pneumonia at various types of facilities revealed that the cumulative incidence of pneumonia was very high $(22.2 \%)$ in the intervention group at rehabilitation hospitals. In addition, the onset of pneumonia was relatively early $(\leq 50$ days after the start of the study) for 3 patients in rehabilitation hospitals. One of these 3 patients was seriously ill and had been taking long-term corticosteroid therapy. Notably, the patients at rehabilitation hospitals included those with obviously serious general health problems and/or immune conditions who could not be admitted to other types of facilities. This fact might have greatly contributed to the observed high incidence of pneumonia in the intervention group at rehabilitation hospitals.

Because they included patients who were seriously ill at baseline, rehabilitation hospitals were excluded from a second analysis. Data from the subjects at "other facilities", including residential care homes, were also excluded from this analysis because all subjects belonged to the control group. Thus, the remaining 2 major facilities of sufficiently large population size with relatively homogeneous patient baseline characteristics (i.e., intensive care homes and nursing care facilities, both for the elderly) were included in a re-analysis of the cumulative incidence of pneumonia for both groups. The re-analysis demonstrated significant efficacy of the present oral care intervention for preventing the onset of pneumonia. The findings suggested that balanced distribution of baseline characteristics between the 2 patient groups should be addressed in the future. 
Secondary Endpoints

Secondary endpoints were analyzed only in subjects who completed all 8 months of the study period with no onset of aspiration pneumonia. The analyses of changes in physical parameters from baseline to the end of the study revealed clear increases in body weight and crural circumference in the intervention group. This finding suggests that the present oral care intervention was useful for preventing sarcopenia in this population.

Among the blood biochemistry parameters, the choline esterase level at month 8 was found to have increased significantly from baseline in the intervention group than in the control group, suggesting a positive nutritional effect of ONS. The peripheral blood lymphocyte count (as an index of immune competence) at month 8 had also increased significantly from baseline in the intervention group, suggesting that the present "wiping" plus "ONS" enhanced immune function, thereby preventing the onset of pneumonia.

\section{Appropriateness of the Inclusion Criteria}

Before the start of the study, the incidence of pneumonia in a high-risk group was estimated to be $\geq 40 \%$ based on pilot investigation of chart, whereas the observed incidence was $\leq 20 \%$ (slightly higher than incidences previously reported $[8,9])$. A re-analysis of only male subjects with dysphagia in the control group showed that the incidence of pneumonia was even higher. Despite the unexpectedly low observed incidence of pneu- monia, the inclusion criteria that had originally been intended to enroll high-risk patients were still considered appropriate.

\section{Conclusion}

The present study suggests that a combination of oral wiping and administering ONS may be effective as a prophylactic intervention against aspiration pneumonia. Based on the preliminary results of this study, we are planning to disseminate this new prophylactic intervention throughout Japan, calling it "wiping plus ONS." The dissemination of wiping plus ONS could prevent the onset or worsening of pneumonia not only in elderly patients with underlying disease but also in elderly people in general (because they are often affected by pre-malnutrition owing to lifestyle choices) and those with sarcopenia. This intervention could reduce the mortality rate from aspiration pneumonia among the elderly.

\section{Acknowledgment}

Our study was supported by a Grant-in-Aid for Scientific Research (FY2013) from the Ministry of Health, Labor and Welfare, Japan.

\section{Disclosure Statement}

The authors declare no conflicts of interest.

\section{References}

1 National Center for Geriatrics and Gerontology (Japan): FY2011 Report of Promotion Program on Health for the Elderly: Eating Disorders and Dysphagia, 2012.

2 Ikeda M, Miki T, Matsuo K, et al: Comparisons of the efficacies of various procedures for removing oral contaminants after oral care: preliminary study in healthy subjects. J Jpn Soc Dysphagia Rehabil 2013;17:233-238.

-3 Ikeda M, Miki T, Atsumi M, Inagaki A, Mizuguchi E, Meguro M, Kanamori D, Nakagawa K, Watanabe R, Mano K, Aihara A, Hane Y, Mutoh T, Matsuo K: Effective elimination of contaminants after oral care in elderly institutionalized individuals. Geriatr Nurs 2014;35: 295-299.

4 Volkert, D, Berner YN, Berry E, Cederholm T, Coti Bertrand P, Milne A, Palmblad J, Schneider S, Sobotka, L, Stanga Z, Lenzen-Grossimlinghaus R, Krys U, Pirlich M, Herbst B, Schütz, T, Schröer W, Weinrebe W, Ockenga
J, Lochs H: ESPEN guidelines on enteral nutrition: geriatrics. Clin Nutr 2006;25:330-360.

5 Stratton RJ, Hébuterne X, Elia M: A systematic review and meta-analysis of the impact of oral nutritional supplements on hospital readmissions. Ageing Res Rev 2013;12:884-897.

-6 Strange I, Bartram M, Liao Y, Poeschl K, Kolpatzik S, Uter W, Siber CC, Stehle P, Volkert D: Effects of a low-volume, nutrient- and energy-dense oral nutritional supplement on nutritional and functional status: a randomized, controlled trial in nursing home residents. J Am Med Dir Assoc 2013;14:628.e1e8.

7 Allen Vl, Methven L, Gosney MA: Use of nutritional complete supplements in older adults with dementia: systematic review and meta-analysis of clinical outcomes. Clin Nutr 2013;32:950-957.

8 Kuzuya M, Kato M; FY2011 Sharing Report of Ministry of Health, Labour and Welfare
Grant-in-Aid for Scientific Research: A Longitudinal Research on Oral Food Intake in Elderly People Requiring Nursing Care, 2012.

9 Kikutani T; FY2009 Sharing Report of the Ministry of Health, Labour and Welfare Promotion Program on Health for the Elderly: System of Providing Oral Care in Persons Admitted to Nursing Care Facilities, 2010.

10 Kuwazawa M, Yoneyama T, Sato Y, Kitagawa $\mathrm{N}$, Imai T, Yamaguchi A, Takeuchi S: Factors associated with the onset of aspiration pneumonia/respiratory tract infection at nursing care facilities. Dent Med Res 2011;31:7-15.

-11 Alan M, Grolimund E, Kutz A, Christ-Crain M, Thomann R, Falconnier C, Hoess C, Henzen C, Zimmerli W, Mueller B, Schuetz P. Clinical risk scores and blood biomarkers as predictors of long-term outcome in patients with community-acquired pneumonia: a 6-year prospective follow-up study. J Intern Med 2015;278:174-184. 